\title{
LPL/AQP7/GPD2 promotes glycerol metabolism under hypoxia and prevents cardiac dysfunction during ischemia.
}

\section{Sohta Ishihama}

Nagoya University

\section{Tatsuya Yoshida}

Nagoya University

\section{Satoya Yoshida}

Nagoya University

Noriyuki Ouchi

Nogoya University Graduate School of Medicine

Yu Mori

Nagoya University

Shunsuke Eguchi

Nagoya University

Teruhiro Sakaguchi

Nagoya University

Takuma Tsuda

Nagoya University

Katsuhiro Kato

Nagoya University

Yuuki Shimizu

Nagoya University

Koji Ohashi

Nogoya University Graduate School of Medicine

Yasuko Bando

Nagoya University Graduate School of Medicine https://orcid.org/0000-0003-4166-6784

Hiroaki Yagyu

Mito Medical Center

Nina Wettschureck

Max-Planck-Institute for Heart and Lung Research

\section{Naoto Kubota}

The University of Tokyo, Graduate School of Medicine

\section{Stefan Offermanns}


Department of Pharmacology, Max Planck Institute for Heart and Lung Research, Bad Nauheim, 61231, Germany https://orcid.org/0000-0001-8676-6805

\section{Takashi Kadowaki}

Gratuate School of Medicine, The University of Tokyo

\section{TOYOAKI MUROHARA}

Nagoya University Graduate School of Medicine

Mikito Takefuji ( $\nabla$ takefuji@med.nagoya-u.ac.jp )

Nagoya University School of Medicine https://orcid.org/0000-0003-4870-7913

\section{Letter}

Keywords: LPL/AQP7/GPD2, glycerol metabolism, cardiac dysfunction, ischemia

Posted Date: December 15th, 2020

DOI: https://doi.org/10.21203/rs.3.rs-81516/v1

License: (c) (1) This work is licensed under a Creative Commons Attribution 4.0 International License. Read Full License 


\section{Abstract}

Fatty acid constitutes a major energy substrate in the heart to fuel contraction under aerobic conditions. Ischemia downregulates fatty acid metabolism to adapt to the limited oxygen supply and makes glucose the preferred substrate. However, the mechanism of the myocardial metabolic shift during ischemia remains unknown. Here, we show that cardiomyocyte secretion of lipoprotein lipase (LPL), a principal enzyme that converts triglycerides to free fatty acids and glycerol, increased during myocardial infarction (MI). Cardiomyocyte-specific LPL deficiency enhanced cardiac dysfunction and apoptosis following MI. Deficiency of aquaporin 7 (AQP7), a glycerol channel in cardiomyocytes, increased the myocardial infarct size and apoptosis in response to ischemia. Ischemic conditions activated glycerol-3-phosphate dehydrogenase 2 (GPD2), which converts glycerol-3-phosphate into dihydroxyacetone phosphate to facilitate ATP synthesis from glycerol. Conversely, GPD2 deficiency exacerbated cardiac dysfunction after acute MI. Together, these results identify that LPL/AQP7/GPD2-mediated glycerol metabolism plays an important role to bridge glucose and lipid metabolism in $\mathrm{Ml}$ and prevent myocardial ischemia-related damage.

\section{Full Text}

Fatty acid constitutes a major energy substrate in the heart to fuel contraction under aerobic conditions ${ }^{1}$. Ischemia downregulates fatty acid metabolism to adapt to the limited oxygen supply and makes glucose the preferred substrate ${ }^{2,3}$. However, the mechanism of the myocardial metabolic shift during ischemia remains unknown. Here, we show that cardiomyocyte secretion of lipoprotein lipase (LPL), a principal enzyme that converts triglycerides to free fatty acids and glycerol ${ }^{4}$, increased during myocardial infarction (MI). Cardiomyocyte-specific LPL deficiency enhanced cardiac dysfunction and apoptosis following MI. Deficiency of aquaporin 7 (AQP7), a glycerol channel in cardiomyocytes ${ }^{5,6}$, increased the myocardial infarct size and apoptosis in response to ischemia. Ischemic conditions activated glycerol-3phosphate dehydrogenase 2 (GPD2), which converts glycerol-3-phosphate into dihydroxyacetone phosphate to facilitate ATP synthesis from glycerol ${ }^{7}$. Conversely, GPD2 deficiency exacerbated cardiac dysfunction after acute MI. Together, these results identify that LPL/AQP7/GPD2-mediated glycerol metabolism plays an important role to bridge glucose and lipid metabolism in $\mathrm{MI}$ and prevent myocardial ischemia-related damage.

The heart has a high rate of ATP demand to sustain contractile activity and maintain tissue perfusion. Approximately $60-90 \%$ of cardiac ATP is produced by the oxidation of fatty acids, whereas the remaining $10-40 \%$ comes from the oxidation of glucose, lactate, ketone bodies and amino acids ${ }^{1}$. Thus, although fatty acids constitute the predominant substrate used in the heart, the cardiac metabolic network is highly flexible with regard to use of other substrates depending on physiological and pathological stress such as exercise, pregnancy, myocardial infarction (MI), and heart failure ${ }^{2}$. The reciprocal relationship between fatty acid and glucose metabolism was first described in the $1960 \mathrm{~s}^{8}$. Specifically, the increased generation of acetyl CoA derived from fatty acid oxidation decreases glucose oxidation in the heart 
through inhibition of pyruvate dehydrogenase, the enzyme that catalyzes pyruvate decarboxylation, a key irreversible step in glucose oxidation ${ }^{9}$. Conversely, increased acetyl CoA generation from glucose oxidation inhibits fatty acid oxidation by inhibiting carnitine palmitoyltransferase 1 , which enhances fatty acid transport into the mitochondria. Ml, which is defined as myocardial cell death owing to prolonged ischemia, remains the leading cause of mortality worldwide ${ }^{10}$. The onset of myocardial ischemia results from an imbalance between oxygen supply and demand. It is generally accepted that under hypoxic conditions, cardiac metabolism shifts from fatty acids to glucose ${ }^{1}$, which is more efficient with respect to ATP production per $\mathrm{O}_{2}$ consumed $^{3}$.

Accumulating evidence suggests that modulating cardiac energy metabolism by increasing glucose oxidation and decreasing fatty acid oxidation, can improve cardiac function in heart disease ${ }^{11}$; however, various factors increase the concentration of plasma free fatty acids (FFA), such as hormonal state in response to myocardial ischemia ${ }^{8}$. This contradiction between the detrimental effects of fatty acid oxidation and increased plasm FFA levels in MI suggests that lipoprotein lipase (LPL), which is the principal enzyme that converts triglycerides in the circulation to FFA ${ }^{4}$, mediates other metabolic pathways in MI. LPL is produced from cardiomyocytes, skeletal muscle and adipose tissue to control local fatty acid uptake ${ }^{12}$, and a genetic study indicates that LPL activation reduces coronary artery disease risk ${ }^{13}$. Glycerol is generated in the process of LPL-catalyzed breakdown of the triglyceride component of lipoproteins to provide fatty acids ${ }^{14}$. Aquaporin 7 (AQP7) is an aquaglyceroporin that facilitates the transport of glycerol across cell membranes into the heart ${ }^{5,6}$. AQP7 deficiency reduces glycerol uptake in the heart and exacerbates pressure overload-induced heart failure ${ }^{15}$, but the role of glycerol as a substrate for energy production in cardiomyocytes under hypoxia conditions remains unclear.

To investigate whether acute MI increases cardiac LPL expression, the left coronary artery in wild-type (WT) mice was ligated. LPL was assayed by immunostaining of sections using an antibody against LPL $1 \mathrm{~h}$ after the ligation (Fig. 1A and 1B). MI increased the intensity of LPL at the capillary endothelium in the infarct area, suggesting that the ischemic conditions enhance LPL expression in vivo. To investigate whether hypoxia conditions affect LPL secretion from isolated adult murine cardiomyocytes, LPL on these cells under 1-h hypoxia conditions was ascertained by immunostaining (Fig. 1C and 1D). Hypoxia conditions significantly increased LPL intensity on the cardiomyocyte surface, suggesting that hypoxia enhanced cardiac LPL secretion. To examine the functional significance of cardiac LPL under ischemic conditions in vivo, we used mice with tamoxifen-inducible cardiomyocyte-specific deficiency for LPL (cmc-LPL KO). Immunostaining analysis revealed decreased LPL in the hearts of cmc-LPL KO mice (Fig. 1E). No differences were observed in hepatic LPL expression between cmc-LPL WT and cmc-LPL KO mice (Figure S1). Notably, cardiomyocyte-specific LPL deficiency suppressed MI-induced LPL expression at the capillary endothelium in the heart, suggesting that LPL is synthesized in cardiomyocytes under ischemic conditions. We performed echocardiography to assess cardiac functionality 1 day after the left coronary artery ligation (Fig. 1F). In control mice, 1-day coronary artery ligation resulted in significantly 
decreased cardiac function, with cardiac LPL deficiency exacerbating the MI-induced cardiac dysfunction. To examine whether cardiac LPL deficiency affects the cardiac apoptosis induced by the ligation, apoptotic cardiomyocytes were assessed by terminal deoxynucleotidyl transferase dUTP nick-end labeling (TUNEL) staining of heart sections 1 day after the ligation (Fig. 1G). LPL deficiency increased MIinduced apoptosis.

As glycerol is produced when LPL converts triglycerides to FFA ${ }^{14}$, we next investigated whether glycerol is a substrate for energy production in cardiomyocytes under hypoxia conditions. Isolated adult murine cardiomyocytes were incubated with glycerol and exposed to hypoxia conditions (Fig. 2A). After 4-h exposure to normoxia or hypoxia, we identified damaged cardiomyocytes using trypan blue vital staining. Glycerol treatment increased cardiomyocyte cell viability already under normoxia conditions in a dosedependent manner; however this effect was enhanced under hypoxia conditions. AQP7 is expressed in adipose tissue, skeletal muscle and the heart, and serves as a glycerol channel ${ }^{15}$. To investigate whether the protective effect of glycerol under cardiac hypoxia requires AQP7, cardiomyocytes were isolated from $\mathrm{AQP7} 7^{+/+}$and $\mathrm{AQP7} 7^{-/-}$mice. AQP7 deficiency decreased glycerol-increased cardiomyocyte cell viability (Fig. 2B). To examine whether AQP7 deficiency increased MI-induced cardiac apoptosis, apoptotic cardiomyocytes were identified by TUNEL staining of heart sections 1 day after the left coronary artery ligation (Fig. 2C and 2D). No difference in apoptosis was observed between AQP7 $7^{+/+}$and AQP7 $7^{-/-}$in control animals whereas AQP7 deficiency significantly increased Ml-induced apoptosis. To determine whether glycerol attenuates the progression of Ml-induced cardiac dysfunction in mice, infarct changes were ascertained in PicroSirius red-stained Sect. 7 days following the ligation (Fig. 2E and 2F). AQP7 deficiency increased the infarct area in the heart. We also performed echocardiography to assess cardiac functionality (Fig. $2 \mathrm{G}$ and $2 \mathrm{H}$ ). In control mice, 7-day $\mathrm{Ml}$ resulted in a significantly decreased fractional shortening, with the response enhanced in $\mathrm{AQP7}^{-/-}$mice. In addition, atrial natriuretic peptide (ANP) in the heart, which is increased upon cardiac dysfunction, was elevated in $\mathrm{AQP7}^{-/-}$mice 7 days following ligation (Fig. 2l).

After the cells capture glycerol, glycerol kinase (GK) catalyzes the phosphorylation of glycerol to yield G3P ${ }^{16}$. GK expression is considered to be restricted to the liver, kidney, and skeletal muscle. To examine $G k$ gene expression in the heart, we performed quantitative polymerase chain reaction (qPCR) analysis of various murine tissues (Fig. 3A), which revealed Gk was also expressed in the heart. In turn, glycerol-3phosphate dehydrogenase 2 (GPD2), which is anchored to the mitochondrial membrane, oxidizes glycerol-3-phosphate (G3P) to dihydroxyacetone phosphate (DHAP) in the cytoplasm ${ }^{7}$. To examine GPD2 enzymatic activity on cardiac mitochondria, mitochondria were isolated from the heart; these exhibited GPD2 activity in vitro (Fig. 3B). Next, as $\mathrm{Ca}^{2+}$ increases GPD2 activity on mitochondria in the liver but not in the brain of rat ${ }^{17}$, cardiac mitochondria were incubated with high concentration of $\mathrm{Ca}^{2+}$. This increased GPD2 activity whereas treatment with EDTA suppressed $\mathrm{Ca}^{2+}{ }^{2+}$ induced GPD2 activation, suggesting that the high $\mathrm{Ca}^{2+}$ concentration enhances GPD2 activity in the heart. To examine whether MI affects GPD2 enzymatic activity in the heart, mitochondria were isolated from the heart $1 \mathrm{~h}$ after sham surgery or coronary artery ligation (Fig. 3C). Ischemic conditions significantly increased the cardiac GPD2 
enzymatic activity. Next, we examined whether glycerol is involved in the energy metabolism in cardiomyocytes. As 4-h hypoxia was shown to decrease cardiomyocyte cell viability (Fig. 2A), we examined intracellular ATP concentrations in isolated adult murine cardiomyocytes under 1-h hypoxia conditions to avoid cell viability effects on ATP production (Fig. 3D). Notably, 1-h hypoxia conditions did not affect cell viability and no difference between control and glycerol treatment was observed. Conversely, 1-h hypoxia significantly decreased ATP production in cardiomyocytes (Fig. 3E). Although glycerol treatment did not increase ATP production under normoxia conditions, glycerol-mediated ATP production was significantly increased under hypoxia conditions. In comparison, the glycerol-dependent ATP production under early hypoxia conditions was suppressed by the GPD2 inhibitor in a dosedependent manner, whereas significant effects on ATP production under normoxia conditions were not detected. To evaluate the role of GPD2 in vivo, we performed coronary artery ligation in $\mathrm{GPD}^{+/+}$and $\mathrm{GPD}^{-/-}$mice. In control mice, cardiac dysfunction was observed at 2 and 4 weeks after the ligation (Fig. 3F). GPD2 deficiency significantly exacerbated MI-induced cardiac dysfunction and increased infarct area in the heart (Fig. 3G).

Here, we demonstrated that glycerol is a substrate for energy production in cardiomyocytes under hypoxia conditions (Fig. $3 \mathrm{H}$ ). Glycerol plays biochemically important roles by serving as the backbone of glyceride lipids ${ }^{18}$. Once glycerol enters the major pathways of carbohydrate metabolism such as glucose metabolism, it also functions as an energy substrate. For example, intracellular G3P and pyruvate are synthesized upon culture of neonatal rat cardiomyocytes with glycerol ${ }^{19}$. In vivo, the use of ${ }^{14} \mathrm{C}$-labeled glycerol reveals that an increase of heart rate in rat induces a concomitant increase in glycerol uptake in the heart ${ }^{20}$.

In particular, the use of glycerol as a fuel is mediated via AQP7 in the heart under pathological situations such as $\mathrm{Ml}$ and heart failure. The expression of AQP7, which is the most prominent aquaglyceroporin in the heart, increases under conditions of altered energy supply such as diabetes mellitus, fasting, exercise, and high-protein diets ${ }^{6}$. Immunohistochemical staining revealed that AQP7 is localized in capillaries of the heart ${ }^{21}$, whereas in vivo experiments using AQP7 ${ }^{-/-}$mice indicated that AQP7 acts as a glycerol channel in cardiomyocytes. Specifically, although AQP7-/- mice exhibit normal cardiac histology and morphology under basal conditions, AQP7 deficiency exacerbates pressure overload-induced cardiac hypertrophy and isoproterenol-induced cardiac dysfunction and increases mortality following pressure overload-induced heart failure ${ }^{15,22}$.

The heart avidly acquires lipids both from circulating FFA and esterified fatty acids bound to lipoproteins 23 , with esterified FFA constituting a major source of cardiac lipids whereas circulating FFA is of minor importance as fuel for the heart. Because the water solubility of FFA is limited, they must undergo esterification with glycerol to form triglycerides, which make up a significant portion of lipoprotein triglycerides in the circulation ${ }^{24}$. LPL is synthesized in parenchymal cells such as adipocytes and cardiomyocytes. GPI-anchored LPL transgenic mice, in which LPL is anchored to cardiomyocytes, are unable to transport LPL from cardiomyocytes to the vascular lumen, suggesting that LPL is produced in 
cardiomyocytes and is transferred to the apical side of endothelial cells, where the enzyme functions in the heart ${ }^{25}$. Moreover, tamoxifen-inducible cardiac LPL deficient mice (MerCreMer LPL ${ }^{\text {flox/flox }}$ ) increase plasma triglyceride concentration and decrease left ventricular fractional shortening 4 weeks after tamoxifen treatment ${ }^{26}$. Thus, in the present study we ligated the coronary artery of cmc-LPL WT and cmc-LPL KO mice 5 days after tamoxifen treatment to avoid the basal cardiac dysfunction caused by LPL deficiency. Our in vivo investigation indicated that MI causes LPL secretion from cardiomyocytes, which in turn prevented Ml-induced cardiac dysfunction. Alternatively, under ischemic conditions, fatty acid translocase translocates away from the sarcolemma in the heart to limit fatty acid uptake ${ }^{27}$. The contradiction between downregulation of fatty acid oxidation and increase of LPL secretion in the heart exposed to Ml supports our model that the upregulation of glycerol metabolism by LPL, which hydrolyzes circulating triglycerides into FFA and glycerol in the vascular lumen, prevents cardiac dysfunction.

Although GPD2 plays an important role in physiological and pathological situations such as the release of insulin in pancreatic islets $\beta$ cells and macrophage inflammatory responses ${ }^{28,29}$, the role of GPD2 in the heart remains unclear. Here, we found that GPD2 deficiency did not affect basal cardiac function and that GPD2 constitutes an important regulator bridging glucose and lipid metabolism in MI. In glycerol metabolism, ATP synthesis with conversion from DHAP to pyruvate, which is oxygen-independent, assists in ATP production under anaerobic conditions. These findings indicated that the use of glycerol as energy source by GPD2-mediated myocardial metabolism is suitable under hypoxia conditions during MI.

Moreover, the correlation between LPL activity and phosphorylation of AMP-activated protein kinase (AMPK), which is an essential component of the adaptive response to cardiomyocyte stress during $\mathrm{Ml}$, is revealed by fasted rats ${ }^{30}$. The intracellular $\mathrm{Ca}^{2+}$ concentration in cardiomyocytes increases under $\mathrm{Ml}$, additionally, $\mathrm{Ca}^{2+}$ is a modulator of GPD2 isolated from rat liver in vitro ${ }^{17}$. We found that $\mathrm{Ca}^{2+}$ activated GPD2 on mitochondria isolated from the heart and that coronary artery ligation increased GPD2 enzymatic activity in vivo. These findings suggested that AMPK and $\mathrm{Ca}^{2+}$ may mediate ATP production from glycerol in MI via LPL and GPD2 activation; however, the mechanism through which glycerol metabolism is activated under ischemia conditions requires further investigation.

In conclusion, our data revealed that Ml-induced LPL secretion from cardiomyocytes increased to prevent myocardial ischemia. AQP7 acts as a glycerol channel in cardiomyocytes during MI. GDP2 causes intracellular glycerol to enter the major pathway of carbohydrate metabolism, glucose metabolism, to produce ATP. The findings that LPL/AQP7/GPD2-mediated glycerol metabolism plays an important role to bridge glucose and lipid metabolism in MI makes this glycerol pathway a promising target for therapeutic intervention in acute Ml.

\section{Declarations}

\section{AUTHOR CONTRIBUTIONS}


S.I., T.Y. and S.Y. performed most of the in vitro and in vivo experiments, analyzed, and discussed data, and commented on the manuscript. Y.M., S.E., T.S., T.T., K.K. and Y.S. performed in vivo experiments and mouse generation. H.Y. generated LPL flox mice. N.W. and S.O. discussed data and generated aMHC-CreERT2 mice. N.K. and T.K. commented on the manuscript and generated GPD2 KO mice. Y.B., K.O., N.O., and T.M. supervised the study and commented on the manuscript. M.T. initiated the study, performed in vitro and in vivo experiments, analyzed and discussed data, and wrote the manuscript.

\section{ACKNOWLEDGMENTS}

We thank the staff from the Division of Experimental Animals at the Nagoya University School of Medicine for assisting with animal experiments. The AQP7 KO mice (RIKEN, RBRC06294) were obtained from Dr. Shimomura (Osaka University). The LPL flox mice generated by Dr. Goldberg (Columbia University) were provided by Dr. Ishibashi and Dr. Takahashi (Jichi Medical University). This work was supported by a Grant-in-Aid for Scientific Research from the Ministry of Education, Culture, Sports, Science and Technology of Japan.

\section{DOMPETING INTERESTS.}

The authors declared no competing interests.

\section{References}

1. Abdurrachim, D., et al. Good and bad consequences of altered fatty acid metabolism in heart failure: evidence from mouse models. Cardiovasc. Res. 106, 194-205 (2015).

2. Kolwicz, S.C., Jr., Purohit, S. \& Tian, R. Cardiac metabolism and its interactions with contraction, growth, and survival of cardiomyocytes. Circ. Res. 113, 603-616 (2013).

3. Fillmore, N., Mori, J. \& Lopaschuk, G.D. Mitochondrial fatty acid oxidation alterations in heart failure, ischaemic heart disease and diabetic cardiomyopathy. Br. J. Pharmacol. 171, 2080-2090 (2014).

4. Young, S.G., et al. GPIHBP1 and Lipoprotein Lipase, Partners in Plasma Triglyceride Metabolism. Cell Metab. 30, 51-65 (2019).

5. Rojek, A., Praetorius, J., Frokiaer, J., Nielsen, S. \& Fenton, R.A. A current view of the mammalian aquaglyceroporins. Annu. Rev. Physiol. 70, 301-327 (2008).

6. Verkerk, A.O., Lodder, E.M. \& Wilders, R. Aquaporin Channels in the Heart-Physiology and Pathophysiology. Int. J. Mol. Sci. 20(2019).

7. Mráč ek, T., Drahota, Z. \& Houštěk, J. The function and the role of the mitochondrial glycerol-3phosphate dehydrogenase in mammalian tissues. Biochim. Biophys. Acta 1827, 401-410 (2013).

8. Lopaschuk, G.D., Ussher, J.R., Folmes, C.D., Jaswal, J.S. \& Stanley, W.C. Myocardial fatty acid metabolism in health and disease. Physiol. Rev. 90, 207-258 (2010). 
9. Doenst, T., Nguyen, T.D. \& Abel, E.D. Cardiac metabolism in heart failure: implications beyond ATP production. Circ. Res. 113, 709-724 (2013).

10. Yeh, R.W., et al. Population trends in the incidence and outcomes of acute myocardial infarction. $N$. Engl. J. Med. 362, 2155-2165 (2010).

11. Gupta, A. \& Houston, B. A comprehensive review of the bioenergetics of fatty acid and glucose metabolism in the healthy and failing heart in nondiabetic condition. Heart Fail. Rev. 22, 825-842 (2017).

12. Kersten, S. Physiological regulation of lipoprotein lipase. Biochim. Biophys. Acta 1841, 919-933 (2014).

13. Khera, A.V., et al. Association of Rare and Common Variation in the Lipoprotein Lipase Gene With Coronary Artery Disease. JAMA 317, 937-946 (2017).

14. Miles, J.M., et al. Systemic and forearm triglyceride metabolism: fate of lipoprotein lipase-generated glycerol and free fatty acids. Diabetes 53, 521-527 (2004).

15. Hibuse, T., et al. The heart requires glycerol as an energy substrate through aquaporin 7 , a glycerol facilitator. Cardiovasc. Res. 83, 34-41 (2009).

16. Brisson, D., Vohl, M.C., St-Pierre, J., Hudson, T.J. \& Gaudet, D. Glycerol: a neglected variable in metabolic processes? Bioessays 23, 534-542 (2001).

17. Beleznai, Z., Szalay, L. \& Jancsik, V. Ca2 + and Mg2 + as modulators of mitochondrial L-glycerol-3phosphate dehydrogenase. Eur. J. Biochem. 170, 631-636 (1988).

18. Lin, E.C. Glycerol utilization and its regulation in mammals. Annu. Rev. Biochem. 46, 765-795 (1977).

19. Gambert, S., Helies-Toussaint, C. \& Grynberg, A. Regulation of intermediary metabolism in rat cardiac myocyte by extracellular glycerol. Biochim. Biophys. Acta 1736, 152-162 (2005).

20. Gambert, S., Helies-Toussaint, C. \& Grynberg, A. Extracellular glycerol regulates the cardiac energy balance in a working rat heart model. Am. J. Physiol. Heart Circ. Physiol. 292, H1600-1606 (2007).

21. Skowronski, M.T., et al. AQP7 is localized in capillaries of adipose tissue, cardiac and striated muscle: implications in glycerol metabolism. Am. J. Physiol. Renal Physiol. 292, F956-965 (2007).

22. Gerdes, A.M. \& Capasso, J.M. Structural remodeling and mechanical dysfunction of cardiac myocytes in heart failure. J. Mol. Cell Cardiol. 27, 849-856 (1995).

23. Goldberg, I.J., Trent, C.M. \& Schulze, P.C. Lipid metabolism and toxicity in the heart. Cell Metab. 15, 805-812 (2012).

24. Chiu, A.P., Wan, A. \& Rodrigues, B. Cardiomyocyte-endothelial cell control of lipoprotein lipase. Biochim. Biophys. Acta 1861, 1434-1441 (2016).

25. Yagyu, H., et al. Lipoprotein lipase (LpL) on the surface of cardiomyocytes increases lipid uptake and produces a cardiomyopathy. J. Clin. Invest. 111, 419-426 (2003).

26. Augustus, A., et al. Cardiac-specific knock-out of lipoprotein lipase alters plasma lipoprotein triglyceride metabolism and cardiac gene expression. J. Biol. Chem. 279, 25050-25057 (2004). 
27. Heather, L.C., et al. Differential translocation of the fatty acid transporter, FAT/CD36, and the glucose transporter, GLUT4, coordinates changes in cardiac substrate metabolism during ischemia and reperfusion. Circ. Heart Fail. 6, 1058-1066 (2013).

28. Eto, K., et al. Role of NADH shuttle system in glucose-induced activation of mitochondrial metabolism and insulin secretion. Science 283, 981-985 (1999).

29. Langston, P.K., et al. Glycerol phosphate shuttle enzyme GPD2 regulates macrophage inflammatory responses. Nat. Immunol. 20, 1186-1195 (2019).

30. Qi, D. \& Young, L.H. AMPK: energy sensor and survival mechanism in the ischemic heart. Trends Endocrinol. Metab. 26, 422-429 (2015).

\section{Figures}


A

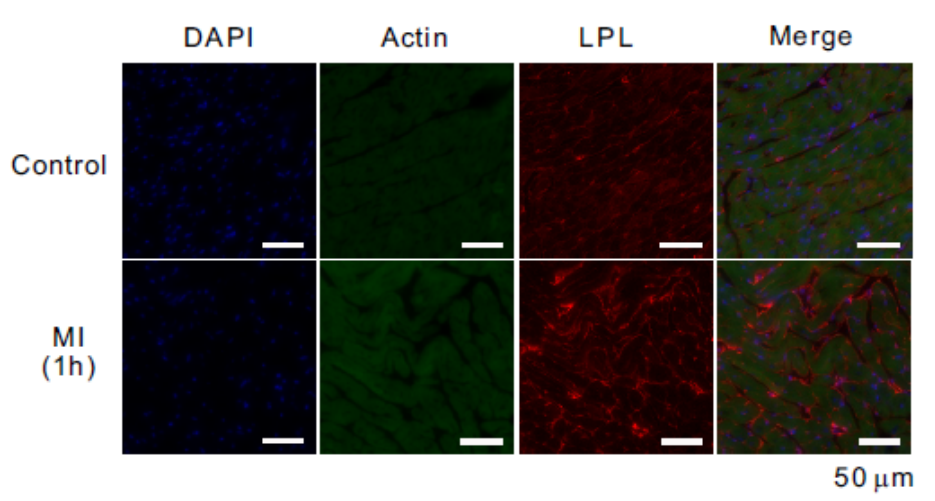

C

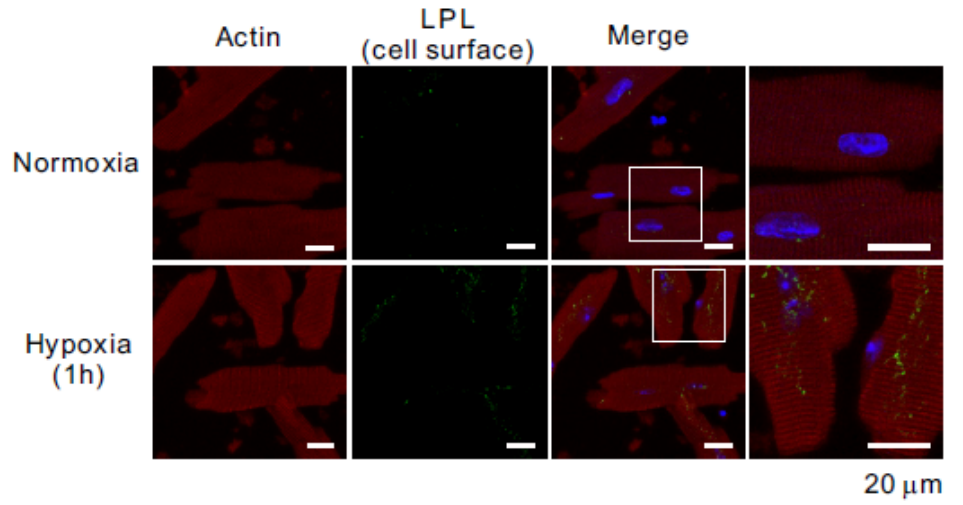

E

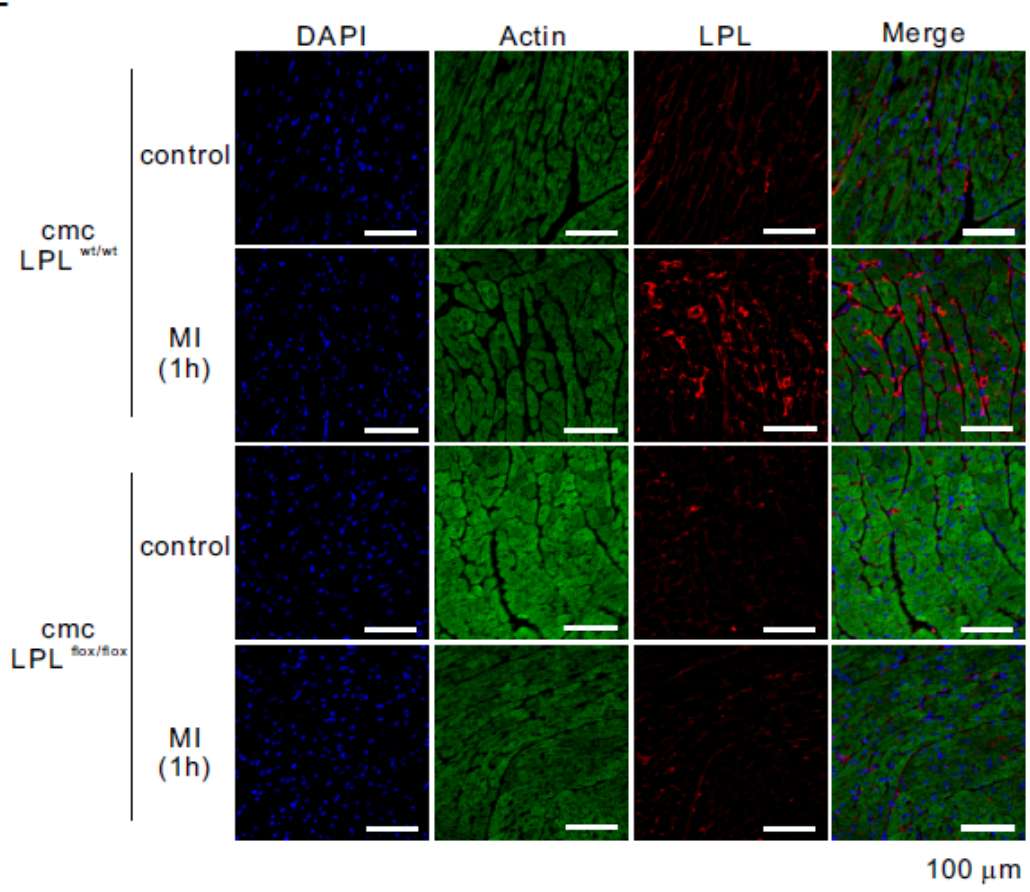

B

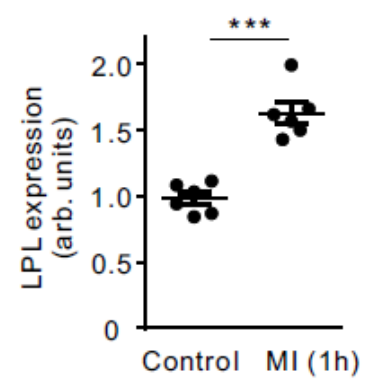

D

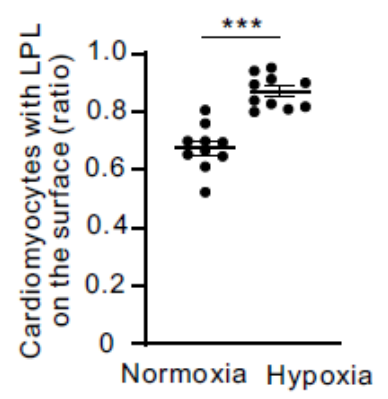

F

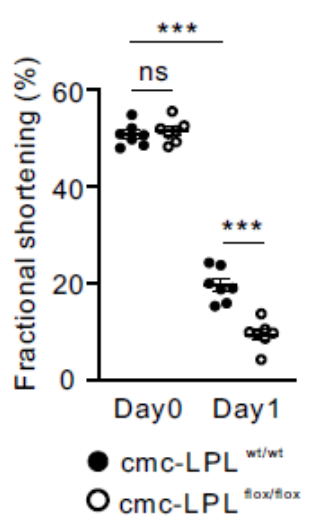

G

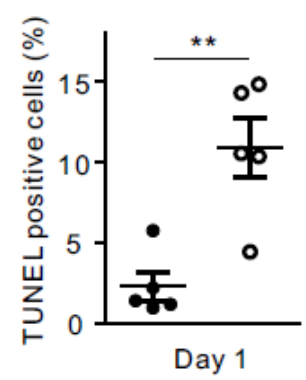

\section{Figure 1}

MI Increases LPL Secretion from Cardiomyocytes. (A) Immunostaining showing LPL expression in the left ventricle after 1-h MI (blue, DAPI; green, actin; red, LPL; scale bar, $50 \mu \mathrm{m}$ ). (B) Statistical evaluation of (A) $(n=6)$. (C) Hypoxia conditions increase LPL expression on the surface of isolated murine cardiomyocytes (red, actin; green, LPL; blue, DAPI; scale bar, $20 \mu \mathrm{m}$ ). Right, magnified image of the boxed area in the panel. (D) Statistical evaluation of $(C)(n=10)$. (E) LPL expression in the left ventricle after 1-h 
MI in cardiac-specific LPLwt/wt and LPLflox/flox mice (blue, DAPI; green, actin; red, LPL; scale bar, 100 $\mu \mathrm{m})$. (F) Left ventricular fractional shortening determined by echocardiogram before and 1 day after coronary artery ligation $(n=7)$. (G) Cardiomyocyte apoptosis as determined by TUNEL assay in the heart $(n=5)$. ns, not significant; ${ }^{* \star}, p<0.01 ; * * \star, p<0.001$. Data are represented as the mean \pm SEM and analyzed with an unpaired Student $t$ test $(B, D$, and $G)$ or 2-way ANOVA followed by the Tukey post hoc test (F).

A

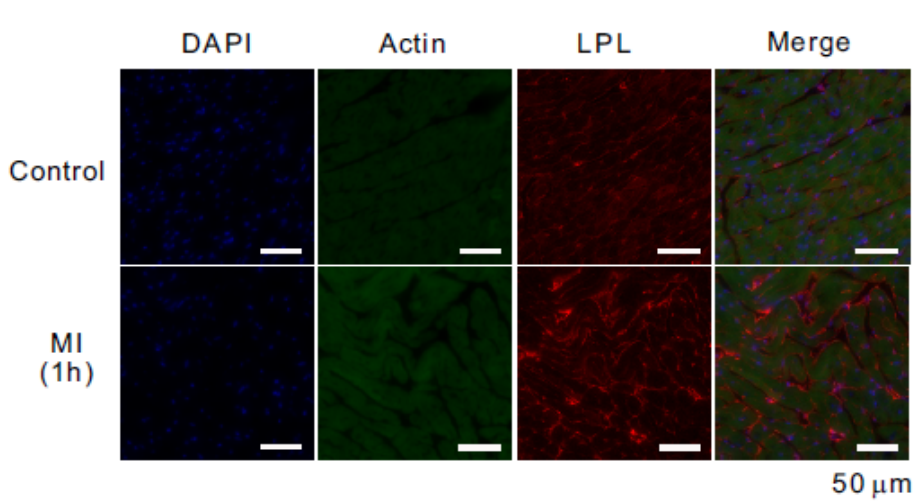

C

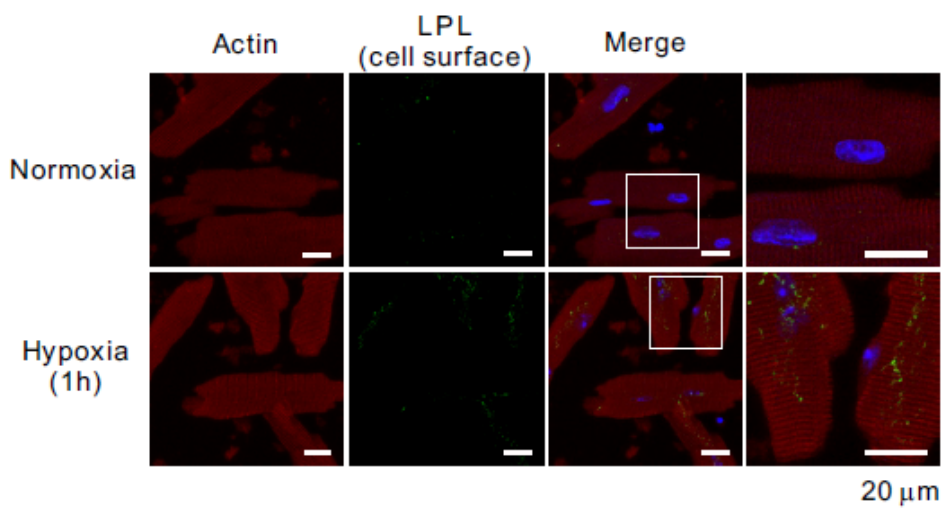

E

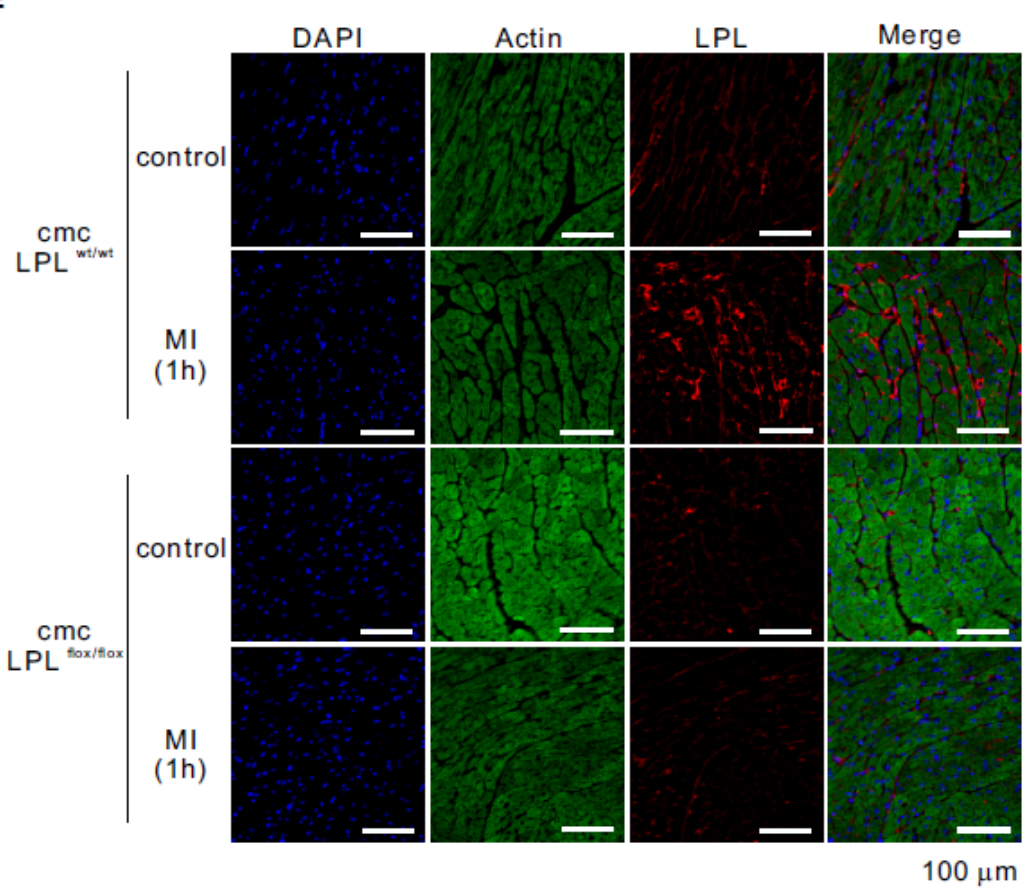

B
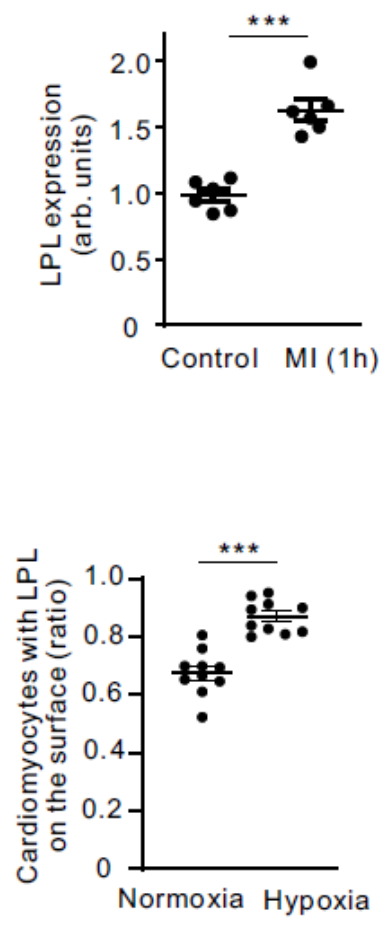

F

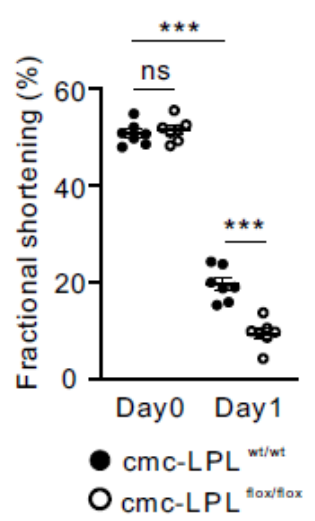

G

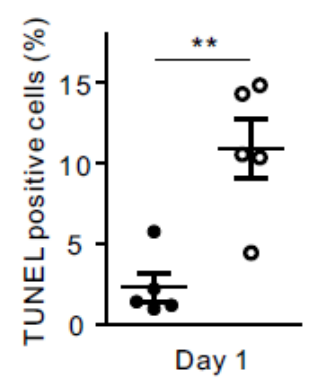

Figure 1 
MI Increases LPL Secretion from Cardiomyocytes. (A) Immunostaining showing LPL expression in the left ventricle after 1-h MI (blue, DAPl; green, actin; red, LPL; scale bar, $50 \mu \mathrm{m}$ ). (B) Statistical evaluation of (A) $(n=6)$. (C) Hypoxia conditions increase LPL expression on the surface of isolated murine cardiomyocytes (red, actin; green, LPL; blue, DAPl; scale bar, $20 \mu \mathrm{m}$ ). Right, magnified image of the boxed area in the panel. (D) Statistical evaluation of $(C)(n=10)$. (E) LPL expression in the left ventricle after 1-h MI in cardiac-specific LPLwt/wt and LPLflox/flox mice (blue, DAPI; green, actin; red, LPL; scale bar, 100 $\mu \mathrm{m})$. (F) Left ventricular fractional shortening determined by echocardiogram before and 1 day after coronary artery ligation $(n=7)$. (G) Cardiomyocyte apoptosis as determined by TUNEL assay in the heart $(n=5) . n s$, not significant; $* \star, p<0.01 ; * \star \star, p<0.001$. Data are represented as the mean \pm SEM and analyzed with an unpaired Student $t$ test $(B, D$, and $G)$ or 2-way ANOVA followed by the Tukey post hoc test (F). 
A

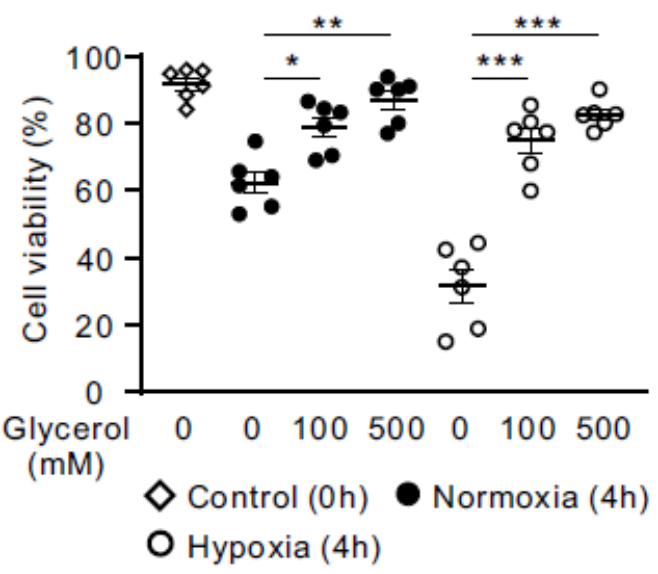

B

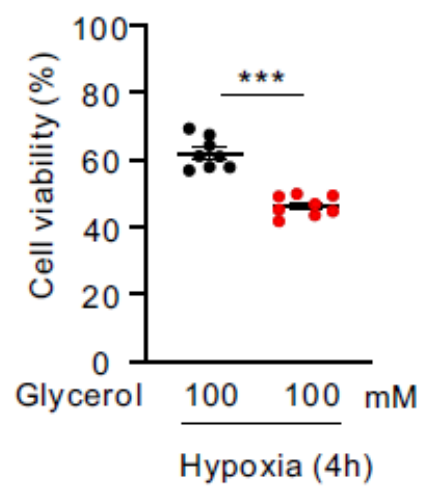

- WT • AQP7 KO

C

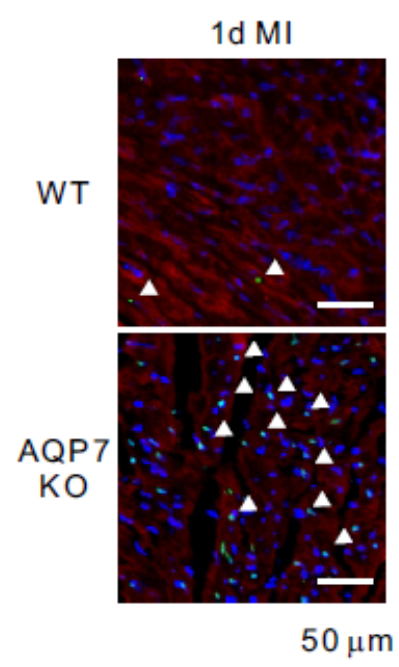

G

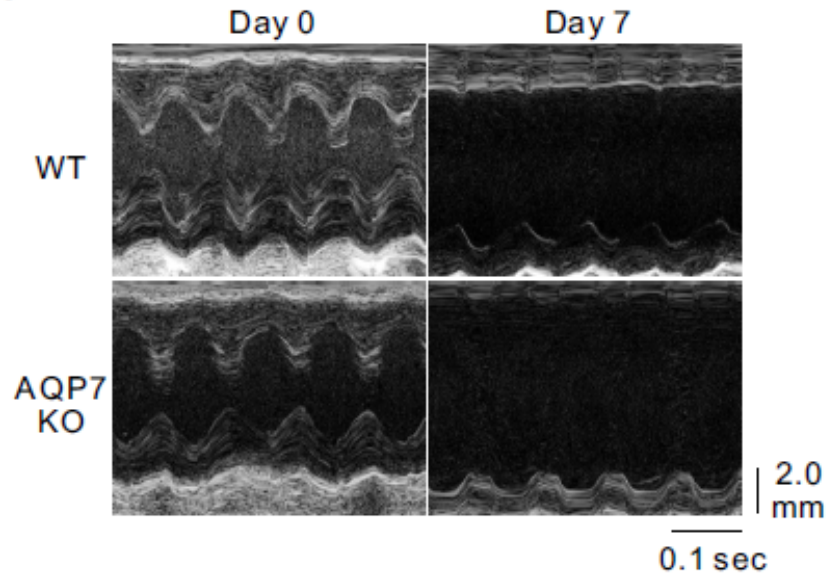

E

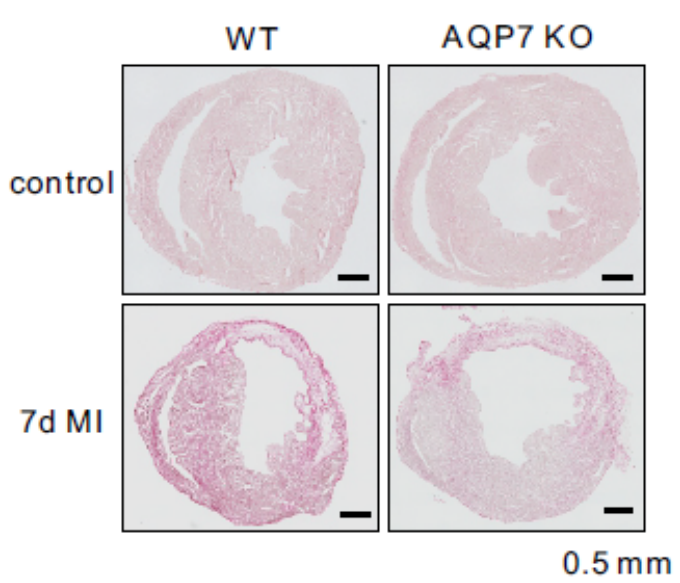

H

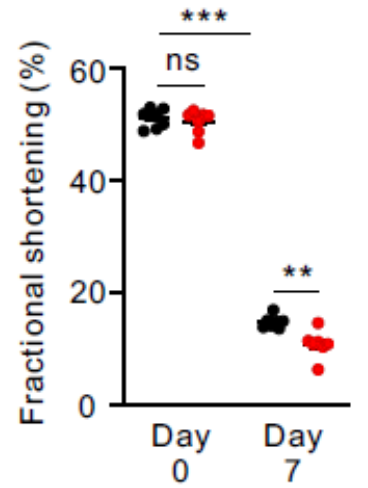

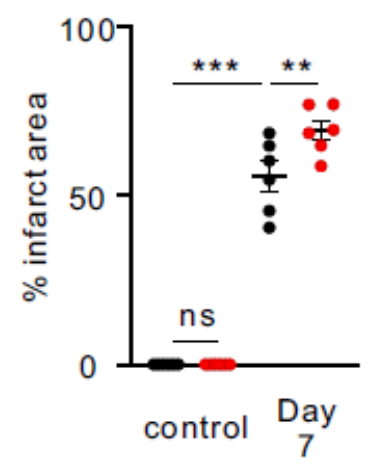

I

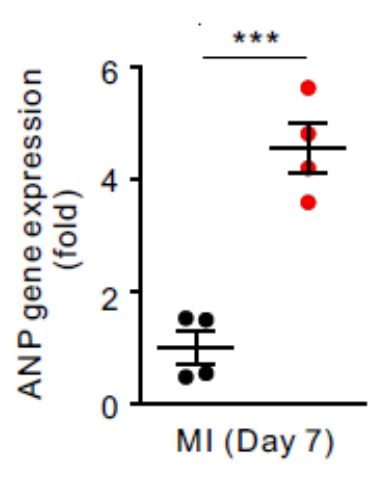

\section{Figure 2}

AQP7 Deficiency Exacerbates Ml-associated Damage in Mice. (A) Cardiomyocyte cell viability in response to hypoxia and glycerol treatment as determined by trypan blue dye exclusion test $(n=4)$. (B) Effect on AQP7 deficiency on cardiomyocyte cell viability $(n=8)$. (C, D) Cardiomyocyte apoptosis (determined by TUNEL assay) in the left ventricle was measured one day after coronary artery ligation $(n=4)$ (red, actin; green, TUNEL; blue, DAPI; scale bar, $20 \mu \mathrm{m})$. (E, F) Infarct area in left ventricles 7 days after MI, as 
determined by PicroSirius red staining $(n=6)$. $(G, H)$ Left ventricular fractional shortening determined by echocardiogram before and 7 days after coronary artery ligation $(n=7)$. (I) Anp gene expression 7 days after coronary artery ligation as determined by qRT-PCR in the heart $(n=4)$. ns, not significant; ${ }^{*}, p<0.05$; $\star *, p<0.01 ; * \star *, p<0.001$. Data are represented as mean \pm SEM and analyzed with an unpaired Student $t$ test (B and I), one-way ANOVA (A), or two-way ANOVA followed by the Tukey post hoc test $(D, F$, and $H)$.

A

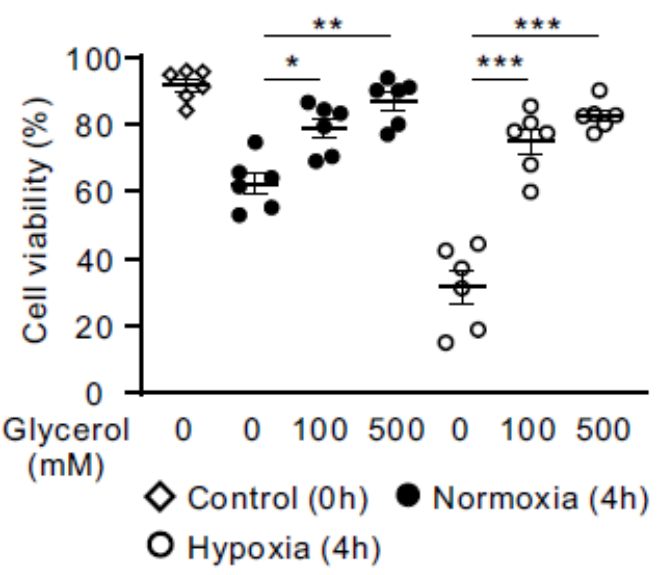

B

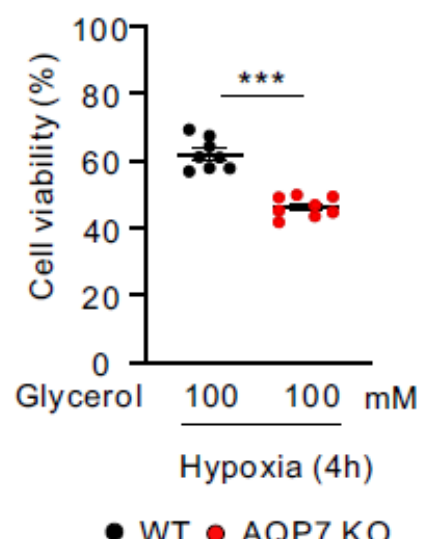

- WT • AQP7 KO

c

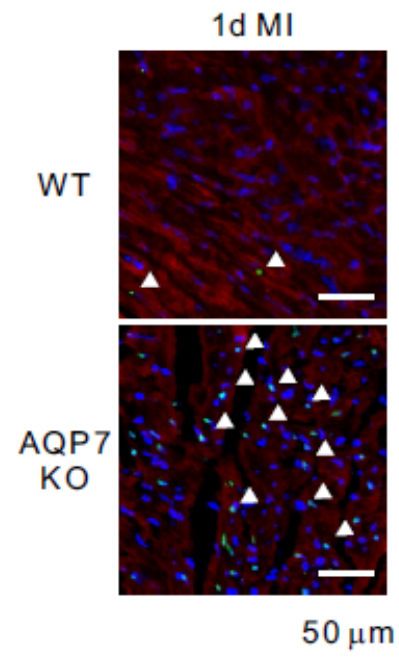

D

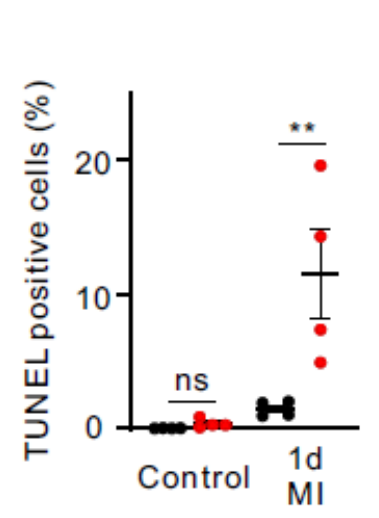

E

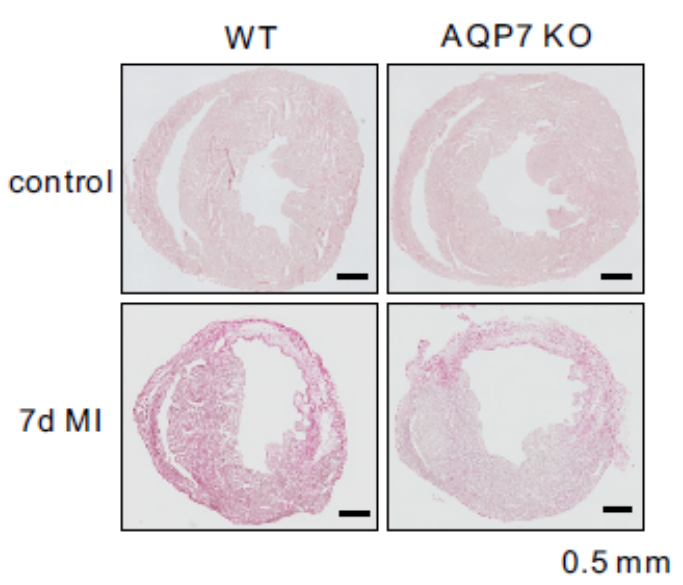

G

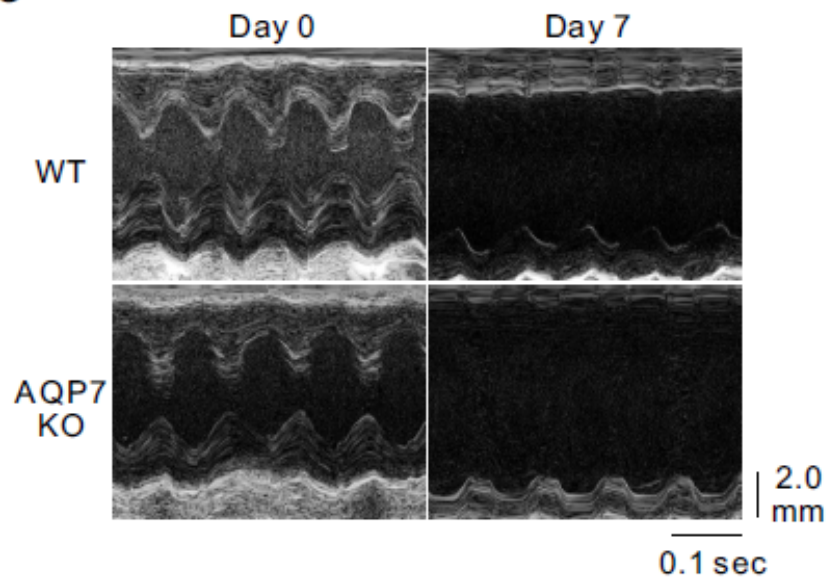

H

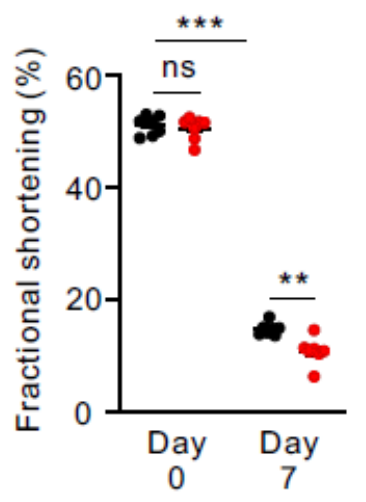

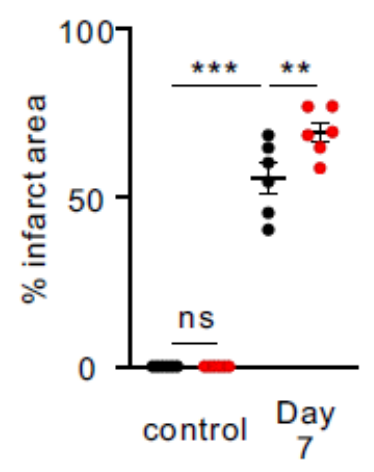

I

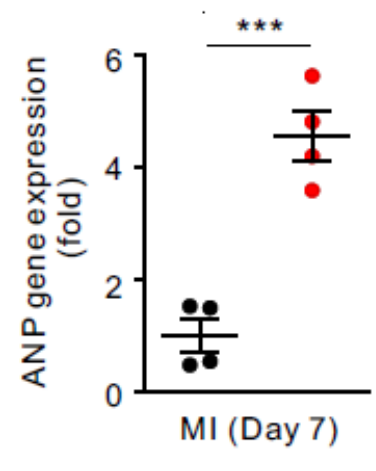

Figure 2 
AQP7 Deficiency Exacerbates Ml-associated Damage in Mice. (A) Cardiomyocyte cell viability in response to hypoxia and glycerol treatment as determined by trypan blue dye exclusion test $(n=4)$. (B) Effect on AQP7 deficiency on cardiomyocyte cell viability $(n=8)$. (C, D) Cardiomyocyte apoptosis (determined by TUNEL assay) in the left ventricle was measured one day after coronary artery ligation $(n=4)$ (red, actin; green, TUNEL; blue, DAPI; scale bar, $20 \mu \mathrm{m}$ ). (E, F) Infarct area in left ventricles 7 days after MI, as determined by PicroSirius red staining $(n=6)$. $(G, H)$ Left ventricular fractional shortening determined by echocardiogram before and 7 days after coronary artery ligation $(n=7)$. (I) Anp gene expression 7 days after coronary artery ligation as determined by qRT-PCR in the heart $(n=4)$. ns, not significant; ${ }^{*}, p<0.05$; $\star \star, p<0.01 ; * \star \star, p<0.001$. Data are represented as mean \pm SEM and analyzed with an unpaired Student $t$ test (B and I), one-way ANOVA (A), or two-way ANOVA followed by the Tukey post hoc test (D, $F$, and $H)$. 
A

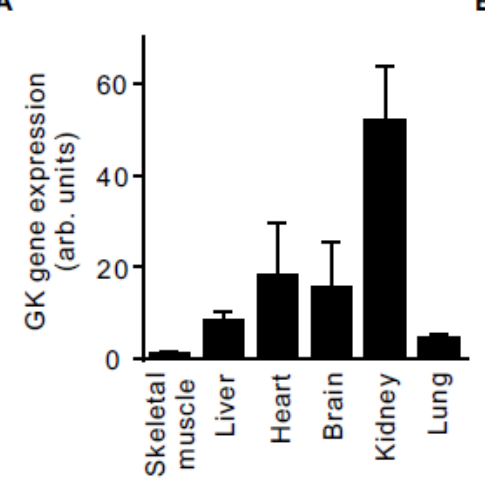

D

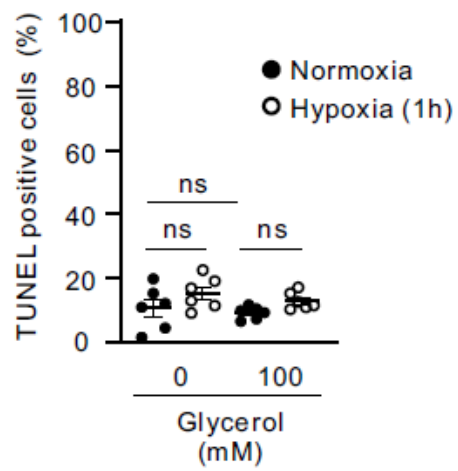

$\mathbf{F}$

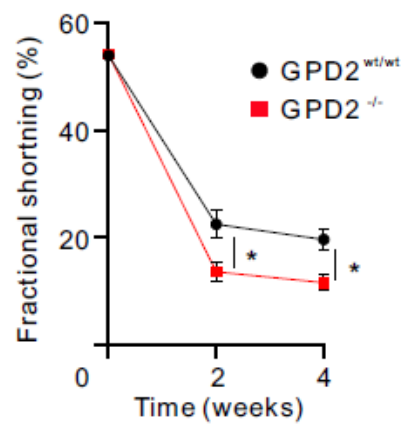

G

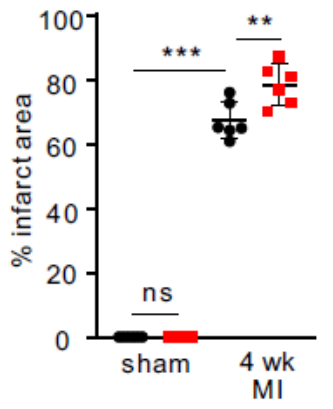

B

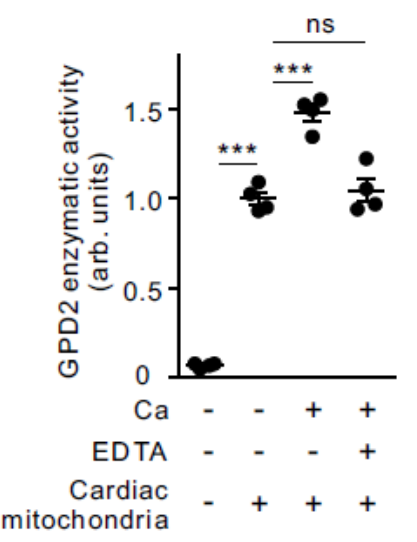

c

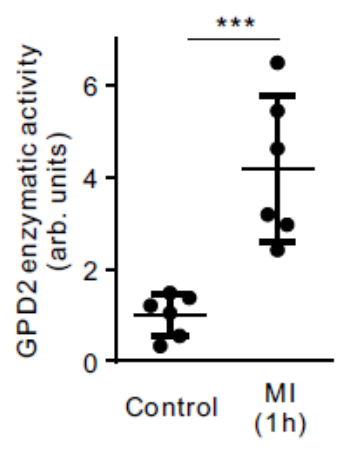

E

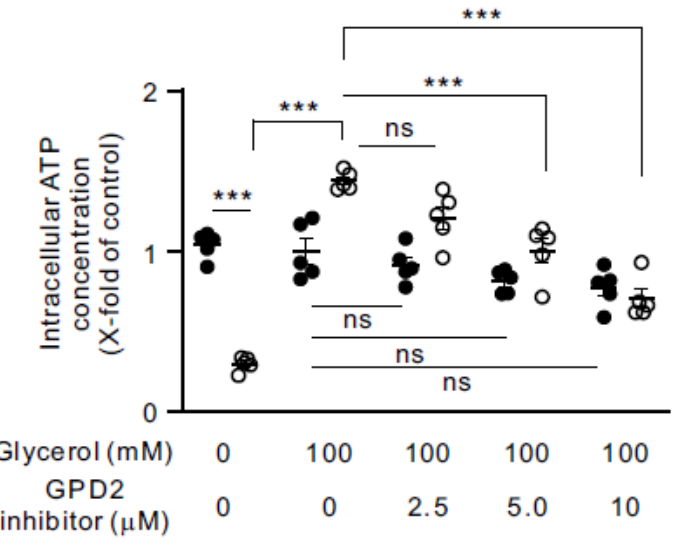

H

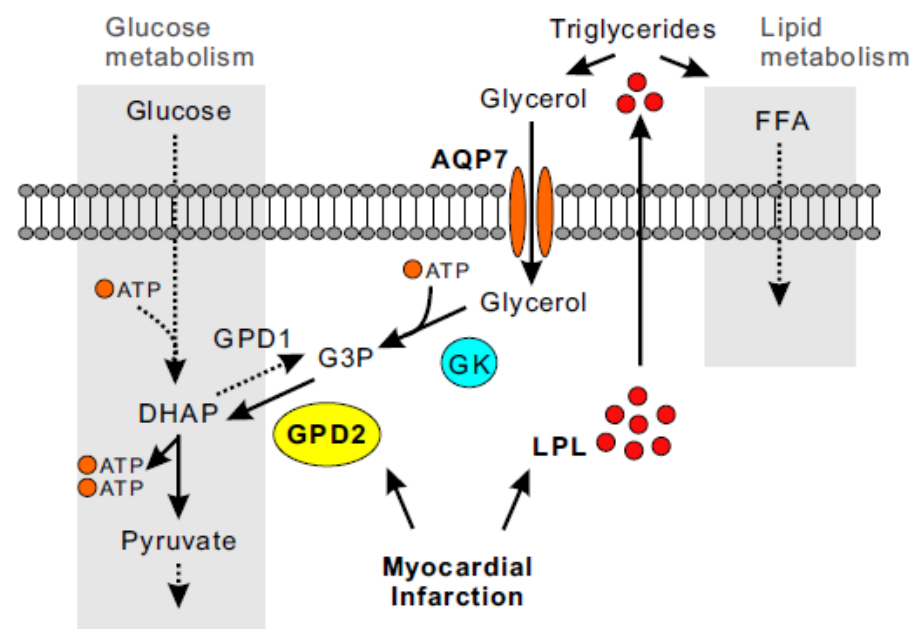

Figure 3

GPD2 Mediates Cardiomyocyte ATP production in Response to Hypoxia Conditions. (A) Gk tissue distribution as assessed by qRT-PCR analysis $(n=4)$. (B) GPD2 enzymatic activity in isolated murine cardiomyocytes $(n=4)$. (C) GPD2 enzymatic activity in the heart $1 \mathrm{~h}$ after the coronary ligation $(n=6)$. (D) Cardiomyocyte apoptosis (determined by TUNEL assay) after 1-h hypoxia conditions $(n=6)$. (E) Intracellular ATP production in isolated murine cardiomyocytes treated with glycerol and a GPD2 inhibitor 
$(n=5)$. (F) Results of echocardiogram analysis of left ventricular fractional shortening performed before, and at 2 and 4 weeks after coronary artery ligation $(n=9)$. (G) Infarct area in left ventricles 7 days after coronary artery ligation, as assessed by PicroSirius red staining $(n=6)$. $(H)$ Schematic diagram showing LPL-, AQP7-, and GPD2-mediated glycerol metabolism activated in response to MI. ns, not significant; *, $p$ $<0.05 ; * \star, p<0.01 ; * \star \star, p<0.001$. Data are represented as mean \pm SEM and analyzed with an unpaired Student $t$ test (C), with 2-way ANOVA followed by the Tukey post hoc test (B, D, and $E$ ) or 2-way repeatedmeasures ANOVA followed by Bonferroni post hoc test ( $F$ and $G$ ).

A

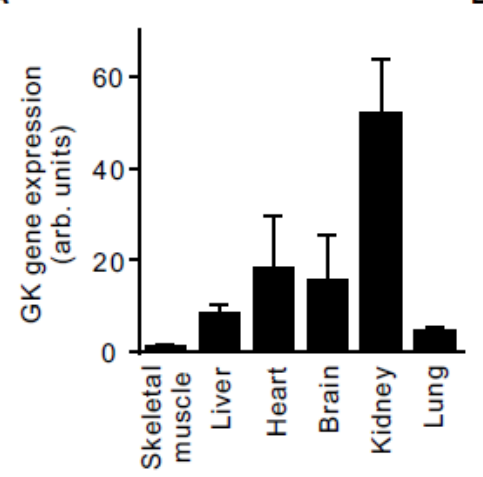

D

F

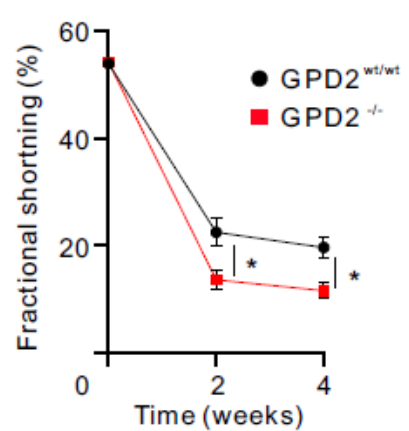

G

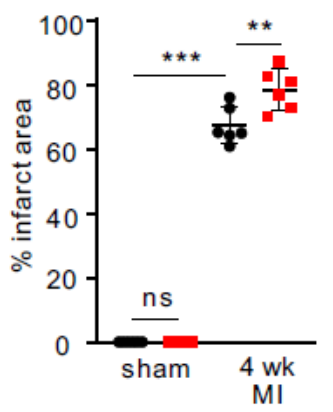

B

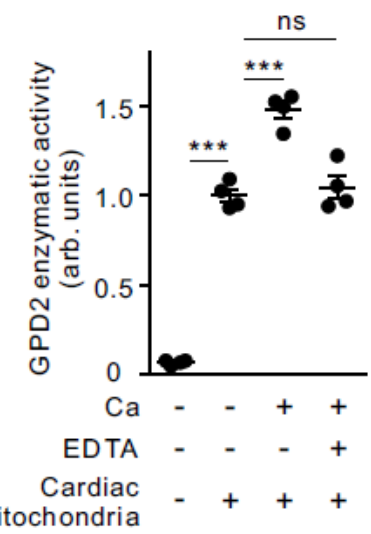

E c

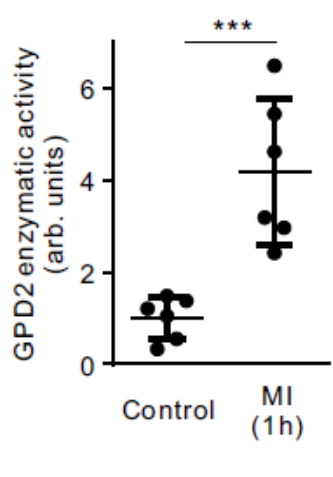

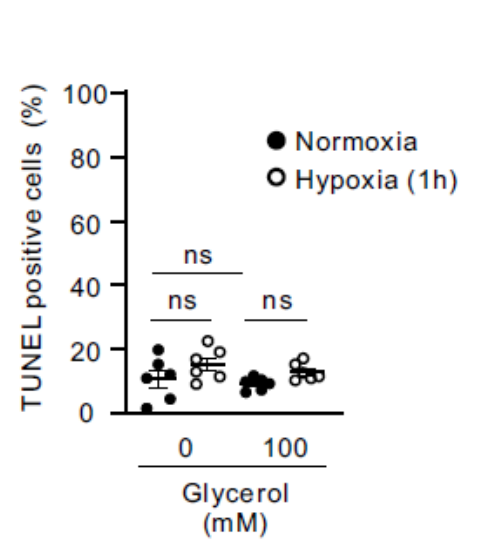

H

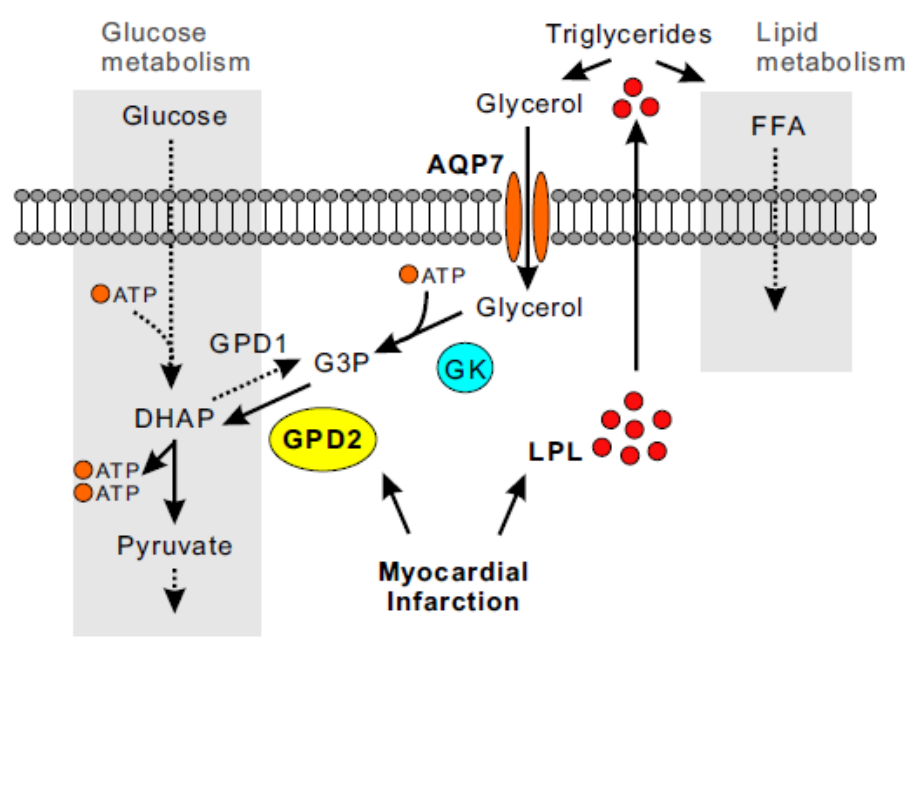




\section{Figure 3}

GPD2 Mediates Cardiomyocyte ATP production in Response to Hypoxia Conditions. (A) Gk tissue distribution as assessed by qRT-PCR analysis $(n=4)$. (B) GPD2 enzymatic activity in isolated murine cardiomyocytes $(n=4)$. (C) GPD2 enzymatic activity in the heart $1 \mathrm{~h}$ after the coronary ligation $(n=6)$. (D) Cardiomyocyte apoptosis (determined by TUNEL assay) after 1-h hypoxia conditions $(n=6)$. (E) Intracellular ATP production in isolated murine cardiomyocytes treated with glycerol and a GPD2 inhibitor $(n=5)$. (F) Results of echocardiogram analysis of left ventricular fractional shortening performed before, and at 2 and 4 weeks after coronary artery ligation $(n=9) .(G)$ Infarct area in left ventricles 7 days after coronary artery ligation, as assessed by PicroSirius red staining $(n=6)$. $(H)$ Schematic diagram showing LPL-, AQP7-, and GPD2-mediated glycerol metabolism activated in response to MI. ns, not significant; *, $p$ $<0.05 ; * \star, p<0.01 ; * \star *, p<0.001$. Data are represented as mean \pm SEM and analyzed with an unpaired Student $t$ test (C), with 2-way ANOVA followed by the Tukey post hoc test (B, $D$, and $E)$ or 2-way repeatedmeasures ANOVA followed by Bonferroni post hoc test ( $F$ and $G$ ).

\section{Supplementary Files}

This is a list of supplementary files associated with this preprint. Click to download.

- Sohtalshihamaetal.MethodsandSuppl.docx

- Sohtalshihamaetal.MethodsandSuppl.docx 